\title{
LaserFIB - the New All-in-one Tool to Speed up Sample Preparation for APT
}

Tobias Volkenandt ${ }^{1}$, Fabián Pérez Willard ${ }^{1}$, Sascha Mueller ${ }^{2}$, Marcus Kaestner ${ }^{2}$ and Benjamin Tordoff ${ }^{1}$

${ }^{1}$ Carl Zeiss Microscopy GmbH, Oberkochen, Baden-Wurttemberg, Germany, ${ }^{2}$ Carl Zeiss SMT GmbH, Oberkochen, Baden-Wurttemberg, Germany

Atom probe tomography (APT) is a powerful technique for highest resolution 3D materials analysis. However, sample preparation for APT is challenging. It is a time-consuming process that involves multiple steps and often different preparation tools which makes it prone to failure and sample loss. With the LaserFIB we present and introduce an all-in-one solution to speed up APT sample preparation and increase its success rate. The LaserFIB features a femtosecond (fs) laser that has been integrated on a ZEISS Crossbeam [1]. This combination enables high-resolution SEM imaging and FIB patterning as well as site-specific sample structuring and preparation using the laser. All laser processing is done in a separate chamber extending the airlock to avoid contamination of the FIB-SEM chamber components (Fig. 1).

In the context of APT sample preparation the laser is used for prepreparation of pillar arrays over an area of hundreds of microns up to centimeters within minutes. Figure 2 shows an exemplary SEM image of such an array with 16 pillars in a titanium alloy (Ti6AlV). The whole array was milled by the laser within 2.5 minutes. The pillars are $100 \mu \mathrm{m}$ in height, with $150 \mu \mathrm{m}$ clearance and feature a tip diameter of $30 \mu \mathrm{m}$ in this case. Achievable tip diameters are in the order of 10 microns, which significantly reduces the necessary time for subsequent final shaping by FIB. The laser also allows to prepare special sample geometries with e.g. handles for tweezers or even custom designed whole coupons. A high success rate is enabled since large numbers of pillars can be prepared within one instrument on the very same sample holder in a streamlined workflow. Due to the integration with a ZEISS Crossbeam, the FIB is readily available and any risk of sample contamination or damage during transfer to other preparation tools is excluded. If required, the system can be equipped with micromanipulators to facilitate an in-situ liftout, too. Given an according sample geometry it is also possible to directly fabricate a complete TEM grid already holding the APT needle out of the sample material. This enables easy investigation by TEM tomography prior to the APT analysis.

With this contribution we will introduce the system and explain the workflow in detail. Application examples for APT sample preparation will be presented, illustrating the capabilities and benefits. 


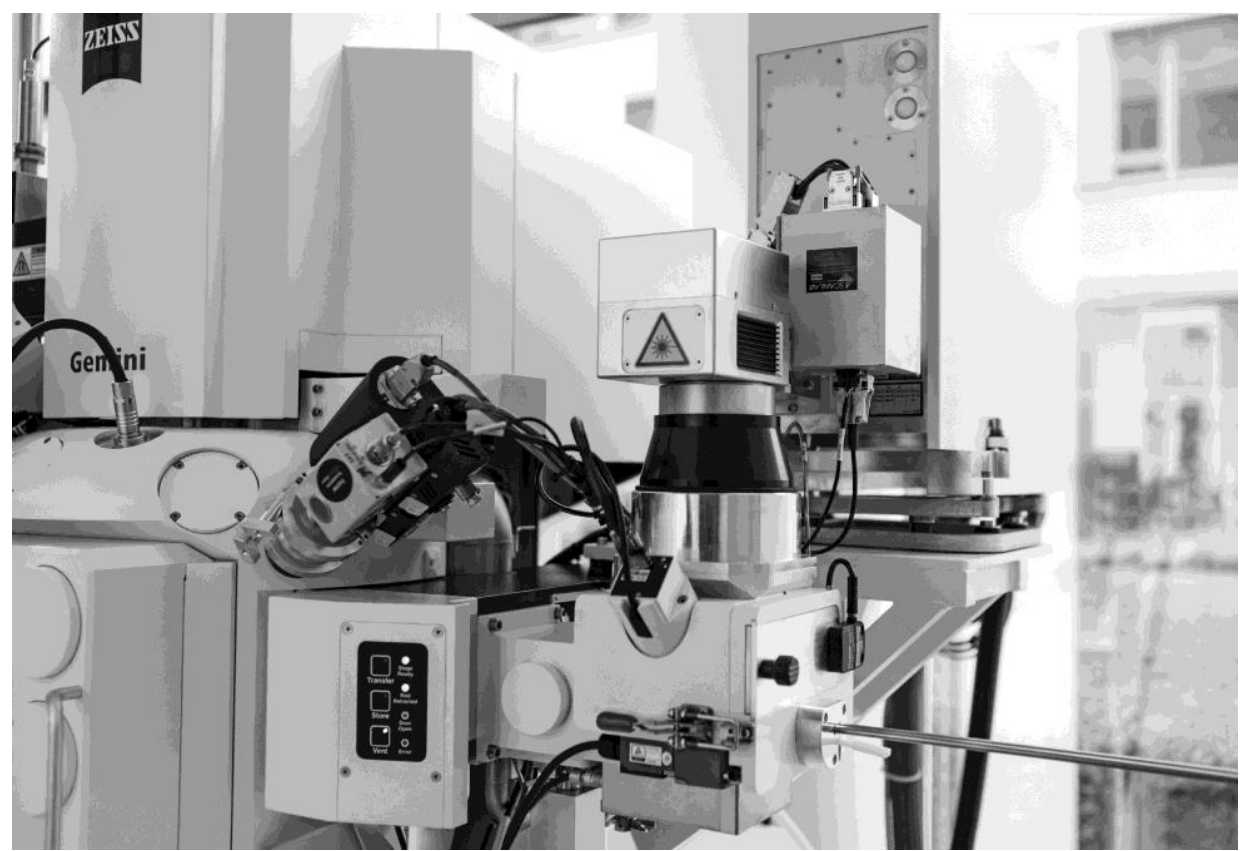

Figure 1. Picture of ZEISS Crossbeam 350 laser with fs laser attached to the airlock.

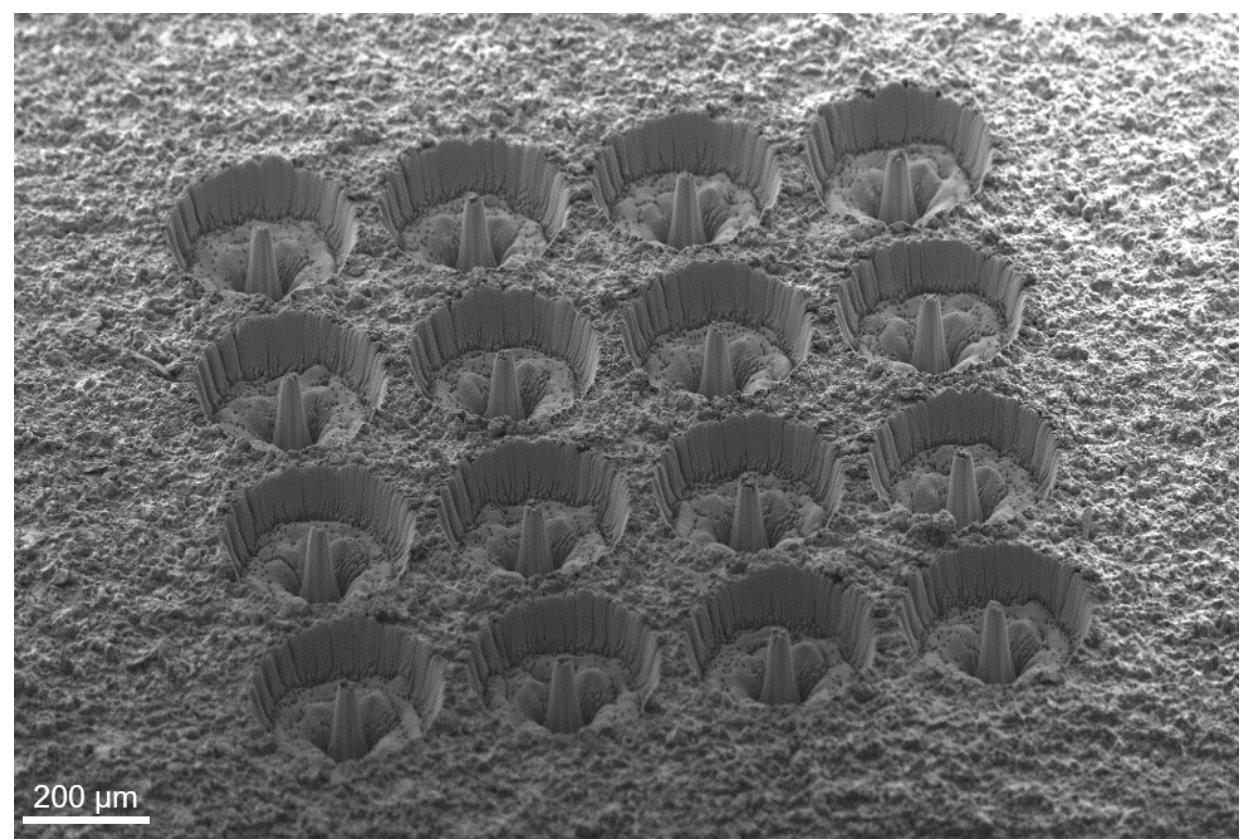

Figure 2. SEM image of an $4 \times 4$ array of laser-prepared pillars in a titanium alloy, ready for further polishing and liftout. The preparation time for the whole array with the laser was only 2.5 minutes. The pillars are $100 \mu \mathrm{m}$ in height, with $150 \mu \mathrm{m}$ clearance and a tip diameter of $30 \mu \mathrm{m}$.

References

[1] https://www.zeiss.com/microscopy/int/products/fib-sem-instruments/crossbeam.html 